\section{Determination of $n$-Alkanes in Mixtures of Saturated Hydrocarbons by Means of Gas Chromatography}

Methods of using molecular sieves $5 \mathrm{~A}$ in gas chromatography for removing $n$-alkanes have been described previously, ${ }^{1,2}$. According to these methods, two tests have to be performed, and the results can only be calculated after correction for differences in the size of samples has been made by means of internal markers. Such a procedure sometimes requires samples larger than those available, for example, for rock-extract analysis.

We have avoided the need for a two-fold test by placing a normal gas-chromatographic column in series with the measuring channel of a heat-conductivity cell, a 10 -cm column filled with Linde $5 \mathrm{~A}$ molecular sieves (50/80 mesh) and the measuring channel of a second heatconductivity cell. This arrangement gives us two chromatograms of a single injected sample, one with and one without the $n$-alkanes. Assuming that all the compounds in the mixture of saturated hydrocarbons have roughly the same heat conductivity, we can calculate the individual $n$-alkane percentage directly from a comparison of the chromatogram areas. These areas can be determined very simply, for example, by being cut out and weighed, provided that the paper used is suitably homogeneous.

An essential condition for the successful functioning of the method is, of course, that the sieves should completely retain all the $n$-alkanes and allow all the isoalkanes and cyclanes to pass. We have found that the activated sieves do indeed retain all the $n$-alkanes, but that they unfortunately also retain a portion of the isoalkanes and cyelanes. Experiments with a number of $\mathrm{C}_{16}$ isomers and 2-Me-eicosane showed that when very small samples were injected $(<1 \mathrm{mg})$ as much as 50 per cent might be retained. Since the percentage always decreased as the amount of sample increased, we came to the conclusion that a certain absolute quantity of isoalkanes is always adsorbed by the sieves. This adsorption can be suppressed to some extent-but not altogether--by coating the sieves with 2 per cent silicone fluid ' $G E / S F$ ' 96 '. Our findings are in agreement with those of Downing et al. ${ }^{3}$. Adlard and Whitham ${ }^{4}$ state that 2-Me-eicosane is not adsorbed from the gas phase. Their experiments were performed with samples weighing several milligrams ${ }^{5}$, however, and with samples as large as this we have not found the adsorption of $\mathrm{C}_{16}$ isomers to be noticeable either.

The equipment can be assembled from various types of commercial gas liquid chromatographic apparatus. The need for two well-matched gas liquid chromatographic detection units is inherent to the method, however. Since two heat-conductivity cells rarely have exactly the same sensitivity, it will usually be necessary to shunt the more sensitive cell electrically.

The method has proved useful for the determination of $n$-alkanes in the $\mathrm{C}_{10}-\mathrm{C}_{20}$ range, where preconcentration is not needed. Above $n-\mathrm{C}_{20}$ preconcentration is required, urea adduction being one method of doing this.

Full details concerning the method and the results obtained with it are shortly to be published elsewhere ${ }^{6}$.

\section{P. A. Schenck \\ E. Eisma}

Koninklijke/Shell Exploratie en Produktie Laboratorium, Rijswijk (Z-H),

The Netherlands.

${ }^{1}$ Brenner, N., and Coates, V. J., Nature, 181, 1401 (1958).

2 Whitham, B. T., Nature, 182, 391 (1958).

${ }^{3}$ Downing, D. T., Kranz, Z. H., and Murray, K. E., Austral. J. Chem., 13, 86 (1960).

4 Adlard, E. R., and Whitham, B. T., Nature, 192, 966 (1961).

'Whitham, B. T. (private communication).

- Proc. Cong. Processes in Organic Geochemistry, organized by the Organic Geochemistry Group (European Branch) of the Geochemical Society, Milan, September 10-12, 1962 (Pergamon Press: in the press).

\section{Sulphitocobalamin}

A WIDE range of cobalamins exist where the cyano group of the original vitamin $\mathrm{B}_{12}$ is replaced, for example, by water (vitamin $\mathrm{B}_{12 b}$ ), halides, nitrite, sulphur-containing groups, and alkyl groups (the coenzymes and analogues) and the nature of the ligand attached to the cobalt has a profound influence on the chemical and physical properties of the cobalamin.

Preliminary experiments directed towards the synthasis of the vitamin $\mathrm{B}_{12}$ coenzyme ${ }^{1}$ consisted of a re-investigation of the action of certain sulphur-containing reducing agents on hydroxocobalamin following the report of Pawelkiewicz ${ }^{2}$, who claimed a synthesis of the coenzyme form of Factor $B$ by the sodium dithionite reduction of cobinamide, Factor $B$, in the presence of adenine. We have repeated this experiment with hydroxocobalamin and failed to obtain any product which contained adenine linked to the cobalt. However, a new lightsensitive product was obtained from the reaction, the same product also being obtained in the absence of adenine. The reaction of sodium bisulphite with hydroxocobalamin also gave the same crystalline product, the spectrum of which was somewhat similar to that of vitamin $\mathrm{B}_{12}$ coenzym $\theta^{3}$. Electrophoresis at $p \mathrm{H} 8$ indicated that the compound contained an additional acidic group comparod with vitamin $\mathrm{B}_{12}$ and that it was stable both in solution and in the solid state in the absence of light. The product contained sulphur and the presence of the monoacidic sulphite ligand was suggested by the electrophoretic behaviour and by the presence of two strong bands at 983 and $1,150 \mathrm{~cm}^{-1}$ in the infra-red spectrum; these bands have been associated with the cobalt-sulphur linked sulphito group by Baldwin ${ }^{4}$ in another series of cobalt-containing complexes. On this basis the sulphito compound is formulated as in the partial structure (I), the remainder of the molecule being the normal cobalamin skeleton.

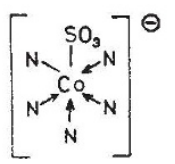

Sulphitocobalamin has been recorded in the earlier literature ${ }^{5}$, but thought to exist only in solutions containing excess sulphite ions. More recently, Hill, Pratt and Williams ${ }^{6}$ have described the properties of solutions of sulphitocobalamin and proposed a structure essentially similar to (I); both these workers and Bernhauer, Renz and Wagner ${ }^{7}$ have described similar sulphito complexes from cobinamide (Factor $B$ ).

Like the coenzyme and its alkyl analogues ${ }^{1,8}$, sulphitocobalamin readily reacts with acid $(p \mathbf{H} 2)$ to form a yellow protonated form (see ref. 6), but the acid solution is unstable and rapidly reverts to hydroxocobalamin. Cyanocobalamin (vitamin $\mathrm{B}_{12}$ ) and hydroxocobalamin (vitamin $\mathrm{B}_{12 b}$ ) require treatment with strong mineral acid to yield the yellow protonated forms.

Aerobic photolysis of an aqueous solution of the sulphito complex gave hydroxocobalamin although under anaerobic conditions $\left(10^{-6} \mathrm{~mm}\right)$ the complex was stable to photolysis. In this respect it resembled the simple alkyl analogues of the coenzyme ${ }^{\theta}$ although the coenzyme itself undergoes fission when subjected to anaerobic photolysis ${ }^{10}$. Treatment of the sulphito complex with eithor potassium cyanide or hydrogen cyanide gives dicyanocobalamin. In this reaction the substitution at the cobalt atom occurs much easier, especially with potassium cyanide, than the similar reaction with the coenzyme and its alkyl analogues.

The course of the reaction of chloramine $T$ with sulphitocobalamin also resembles the corresponding reactions with the coenzyme and the alkyl cobalamins, and differs in 